\title{
Study on the medication rule of traditional Chinese medicine in the treatment of acute pancreatitis based on machine learning technology
}

\author{
Wei-Wei Lu $^{1,2 \#}$, Xi Chen ${ }^{3 \#}$, Jian-Li Ni ${ }^{4,5 \#}$, Wen-Jie Cai ${ }^{6}$, Sheng-Liang Zhu ${ }^{1}$, Ai-Hua Fei ${ }^{2}$, Xiao-Su Wang ${ }^{1}$ \\ ${ }^{1}$ Department of Gastroenterology, Yueyang Hospital of Integrated Traditional Chinese and Western Medicine, Shanghai University of Traditional \\ Chinese Medicine, Shanghai, China; ${ }^{2}$ Department of General Practice, Xinhua Hospital, Shanghai Jiaotong University School of Medicine, \\ Shanghai, China; ${ }^{3}$ Department of Emergency, Zhejiang Provincial People's Hospital, Affiliated People's Hospital, Hangzhou Medical College, \\ Hangzhou, China; ${ }^{4}$ Department of Traditional Chinese Medicine, Chongming Branch Hospital Affiliated to Xinhua Hospital, Shanghai Jiao Tong \\ University School of Medicine, Shanghai, China; ${ }^{5}$ Department of Traditional Chinese Medicine of Xinhua Hospital, Shanghai Jiao Tong University \\ School of Medicine, Shanghai, China; ${ }^{6}$ School of Medical Instrument and Food Engineering, University of Shanghai for Science and Technology, \\ Shanghai, China \\ Contributions: (I) Conception and design: WW Lu, X Chen, JL Ni, XS Wang; (II) Administrative support: SL Zhu, AH Fei, XS Wang; (III) Provision \\ of study materials or patients: WW Lu, X Chen; (IV) Collection and assembly of data: X Chen, JL Ni, WJ Cai; (V) Data analysis and interpretation: \\ WW Lu, AH Fei, XS Wang; (VI) Manuscript writing: All authors; (VII) Final approval of manuscript: All authors. \\ \#These authors contributed equally to this work. \\ Correspondence to: Xiao-Su Wang. Department of Gastroenterology, Yueyang Hospital of Integrated Traditional Chinese and Western Medicine, \\ Shanghai University of Traditional Chinese Medicine, Shanghai, China. Email: xswangxs0084@163.com; Ai-Hua Fei. Department of General \\ Practice, Xinhua Hospital, School of Medicine, Shanghai Jiao Tong University, Shanghai 200092, China. Email: feiaihua@xinhuamed.com.cn.
}

Background: To analyze the rule of traditional Chinese medicine in the treatment of acute pancreatitis (AP). Methods: Using machine learning technology and artificial intelligence, we collected 516 traditional Chinese medicine compounds for treating AP in the recent past 20 years, and analyzed the application of Chinese medicine in the field of AP. The data set was established by the ingredients of each prescription and its corresponding effectiveness. $90 \%$ of the data was divided into the training set, and the remaining $10 \%$ of the data was used as the test set. We employed random forest method to build a model to predict the efficacy of the prescriptions in the treatment of AP. The R-squared score and mean absolute error was used to evaluate the model's performance.

Results: The most frequently used drugs were rhubarb, Radix Bupleuri, Fructus Aurantii Immaturus, and Mirabilite. Rhubarb and Rhizoma Corydalis had the greatest curative effect. The random forest model that fit all data showed that its R-squared score reached 0.8021. And the results predicted on the test set showed that the R-squared score reached 0.7318 .

Conclusions: Soothing the liver, promoting qi, clearing heat, removing obstructions of organs, activating blood, and resolving stagnation are the treatment methods for AP.

Keywords: Acute pancreatitis (AP); traditional Chinese medicine; artificial intelligence

Submitted Aug 05, 2021. Accepted for publication Oct 16, 2021.

doi: 10.21037/apm-21-2505

View this article at: https://dx.doi.org/10.21037/apm-21-2505

(c) Annals of Palliative Medicine. All rights reserved. 


\section{Introduction}

Acute pancreatitis (AP) is a common acute abdominal disease with increasing morbidity year by year, and the mortality rate of severe AP is notably high. A large number of studies have shown that the clinical efficacy of treating AP with the combination of traditional Chinese and Western medicine can be significantly improved. Chinese medicine is characterized by multiple targets, multiple pathways, strong integrity, and few toxic side effects, which has many advantages in the treatment of AP. It can inhibit the expression of inflammatory factors, significantly relieve clinical symptoms, effectively shorten the course of the disease, restore gastrointestinal function as early as possible, reduce complications, and improve the cure rate. In Chinese traditional medicine, a compound prescription is a group of drugs that are properly combined by selecting the appropriate drugs and their dosage after the treatment method has been determined by identifying the cause. By analyzing the dosing rules in different traditional Chinese medicine compounds, it is favorable to explore the effectiveness of the drugs and provide a basis and new ideas for the precise traditional Chinese medicine treatment of acute pancreatitis. Artificial intelligence technology can find correlations between data in the vast amount of Chinese medicine big data, discover potential empirical patterns in Chinese medicine treatment, and continue to innovate and develop on the basis of existing human experience. At the same time, analysis of the dosing rules of Chinese medicine in the treatment of acute pancreatitis can provide a basis for accurate analysis of the efficacy of combined Chinese and Western medicine treatment, which is a subject we need to further study.

Artificial intelligence (AI) has developed rapidly in recent years, and it has played a huge role in medicine, transportation, military and other fields. The most popular methods include artificial neural networks, Bayesian networks, decision trees, random forests, support vector machine (SVM), deep learning, and etc. In the field of Traditional Chinese Medicine (TCM), AI technology has made great achievements in symptom analysis, syndrome differentiation analysis, herb elements analysis and efficacy analysis (1). For example, Zhang et al. developed a recurrent neural network model to detect 187 diseases and achieved the accuracy of $80.5 \%$ (2). AI-assisted inspection, olfaction and auscultation, interrogation and palpation help get objective and quantitative syndrome differentiation which might contribute to the promotion of TCM (3). The drug-like properties of Chinese herbal compounds can be predicted with machine learning methods (4). Wang et al. used network embedding and deep learning to predict TCM side-effect and achieved the accuracy of $90.8 \%$ (5). However, few studies have used AI to analyze the treatment efficacy of TCM. Recently, Xie et al. proposed a novel association learning framework which integrated data mining methods, statistical tests and TCM knowledge to find the most effective herbs for patients with rheumatoid arthritis (6). To the best of our knowledge, there is no study that utilize AI to reveal the rules of TCM prescription medication for acute pancreatitis.

Our group used artificial intelligence and machine learning technology to collect 516 traditional Chinese medicine compounds for the treatment of AP from the China National Knowledge Internet (CNKI) database over the past 20 years, and discovered the medication rule among them. The report is below.

\section{Source}

By searching the China Knowledge Network, 516 traditional Chinese medicine compound prescriptions for the treatment of AP from 1999 to 2019 were collected and organized, which were prescribed according to traditional Chinese medicine diagnosis and typing, and were complete in taste, accurate in dosage, and had definite efficacy after randomized trials. In total there were 157 traditional Chinese medicines. A standardized database was established.

\section{Methods}

\section{Data preparation and input}

The names of the drugs in the compound were standardized referring to the Handbook Chinese Herbal Medicine (7) and then inputted to create a database. Table 1 shows 5 of the prescriptions as well as the number of patients and efficiency rates reported in the literature, 1 means that this Chinese medicine is included in the prescription, and 0 means that this medicine is not included. The efficacy classifications of drugs are referenced in the Chinese Herbal Medicine and Chinese Materia Medica (8).

\section{Frequency of single drug use}

The frequency of the use of a single drug was calculated according to the formula $\mathrm{Yi}=\mathrm{Ni} / \mathrm{M}$. Yi is the frequency 
Table 1 Chinese medicine prescription database

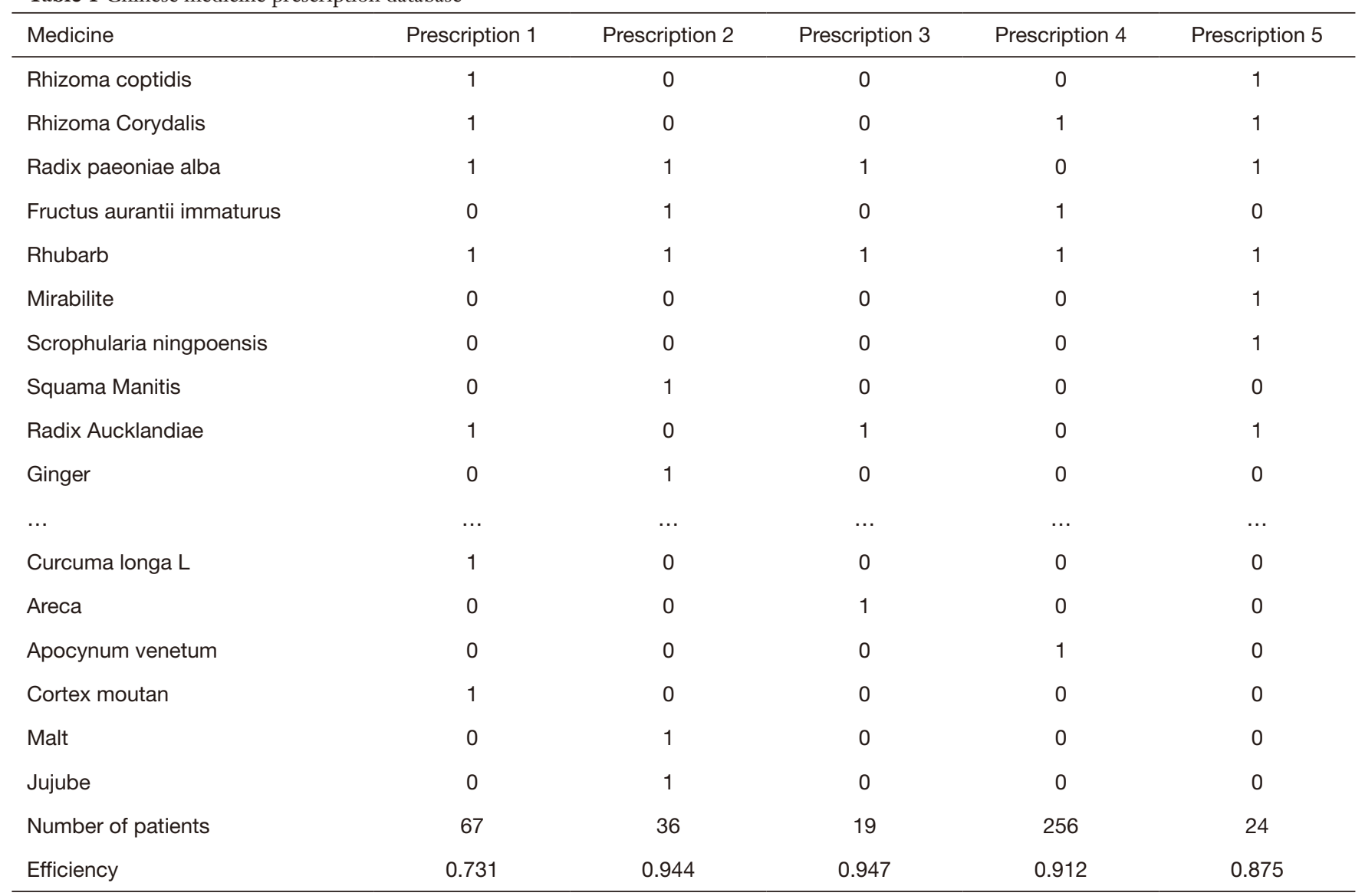

of the use of category i traditional Chinese medicine (\%), $\mathrm{Ni}$ is the number of uses of category i traditional Chinese medicine, and $\mathrm{M}$ is the total number of traditional Chinese medicine compounds. The frequencies of the use of the top 18 herbal medicines are listed in Table 2 .

\section{Random forest model}

The random forest algorithm is an effective machine learning algorithm that integrates multiple decision trees to improve accuracy through integrated learning, and can be used to study classification and regression problems. The random forest algorithm takes into account the interaction of variables, considers the influence of various factors on the results, and scores the importance of the results (9).

The first part of the random forest algorithm involved modeling all the data to explore the medication rule of different drugs in the treatment of AP.

In the second part of the random forest model, $90 \%$ of the data was used for modeling training, and $10 \%$ of the data was used for testing and evaluation. The evaluation of the unknown data reflects the reliability of the model and is generally more valuable for practical evaluation.

\section{Statistical analysis}

The evaluation indicator was expressed as R-squared score (decision factor):

$$
R^{2}=1-\frac{\sum\left(Y_{-} \text {actual }-Y_{-} \text {predict }\right)^{2}}{\sum\left(Y_{-} \text {actual }-Y_{-} \text {mean }\right)^{2}}
$$

The analysis was performed according to the presence or absence of the drug in the prescription, regardless of the dose.

\section{Results}

\section{Frequency of single drug use}

The top 4 most frequently used herbal medicines were 
Table 2 Frequency of single drug use

\begin{tabular}{|c|c|c|}
\hline Traditional Chinese medicine & $\begin{array}{l}\text { Number of } \\
\text { uses } \mathrm{Ni}\end{array}$ & $\begin{array}{c}\text { Frequency } \\
\text { of use Yi (\%) }\end{array}$ \\
\hline Rhubarb & 498 & 96 \\
\hline Radix Bupleuri & 399 & 77 \\
\hline Fructus aurantii immaturus & 369 & 71 \\
\hline Mirabilite & 363 & 70 \\
\hline Magnolia officinalis & 314 & 60 \\
\hline Scutellaria baicalensis Georgi & 299 & 57 \\
\hline Radix paeoniae alba & 271 & 52 \\
\hline Rhizoma Corydalis & 241 & 46 \\
\hline Radix Aucklandiae & 230 & 44 \\
\hline Pinellia ternata & 112 & 21 \\
\hline Rhizoma coptidis & 101 & 19 \\
\hline Glycyrrhiza uralensis & 98 & 18 \\
\hline Radix Paeoniae rubra & 94 & 18 \\
\hline Salvia miltiorrhiza & 91 & 17 \\
\hline Dandelion & 83 & 16 \\
\hline Gardenia jasminoides Ellis & 73 & 14 \\
\hline Rhizoma picrorhizae & 70 & 13 \\
\hline Semen Persicae & 69 & 13 \\
\hline
\end{tabular}

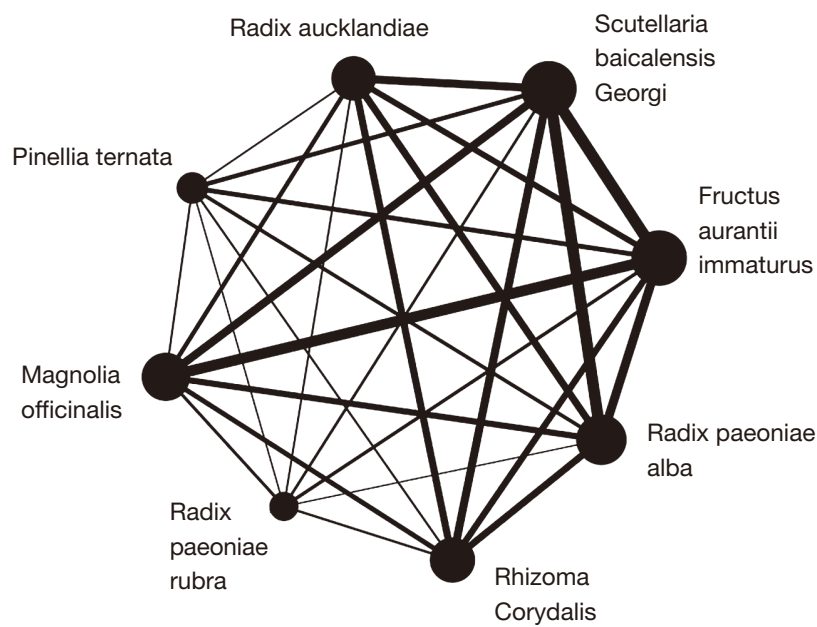

Figure 1 Analysis of the association between the frequency of single drug use. The size of the circle represents the number of times the medicine appears in the prescription and the thickness of the line represents the number of times the two medicines appear together in the prescription. rhubarb, Radix Bupleuri, Fructus Aurantii Immaturus, and Mirabilite (see Table 2).

In Figure 1 we have selected a total of 8 drugs that ranked in the top 10 in importance and also appeared more than 90 times in the prescriptions, to show the frequency of single drug use in Chinese compound prescriptions and their association.

\section{Random forest model}

The random forest model was built with scikit-learn package in Python. The hyperparameter max_depth which means the maximum depth of the decision tree was set to 20. The hyperparameter n_estimators which indicates the number of trees in the forest was set to 100 . Parameter max_depth=20, n_estimators $=100$. The model was trained and the determination coefficient $\mathrm{R}^{2}$ reached 0.8021 . The effect of different traditional Chinese medicine ingredients on the efficacy of the treatment of AP was analyzed according to this model. The first 50 of these drugs are ranked as follows:

Rhubarb, Rhizoma Corydalis, Pinellia Ternata, Magnolia Officinalis, Radix Paeoniae Alba, Radix Bupleuri, Scutellaria Baicalensis Georgi, Curcuma Longa L, Fructus Aurantii Immaturus, Radix Aucklandiae, Semen Persicae, Salvia Miltiorrhiza, Rhizoma Coptidis, Glycyrrhiza Uralensis, Rhizoma Picrorhizae, Mirabilite, Dandelion, Gardenia Jasminoides Ellis, Radix Astragal, Radix Gentiana, Aspongopus Chinensis Dallas, Radix Rehmanniae, Safflower, Cortex Moutan, Flos Lonicerae, Paeoniae Radix, Mirabilitum Dehydratum, Forsythia Suspensa, Curcuma Aromatica, Areca, Artemisiae Capillaris, Fructus Meliae Toosendan, Sargentgloryvine Stem, Cortex Phellodendri, Massa Medicata Fermentata, Aurantii Nobilis Pericarpium, Semen Raphani, Asarum, Patrinia Scabiosaefolia Fisch, Herba Plantaginis, Euphorbia Kansui, Sanguisorba Officinalis L, Caulis Perillae, Lysimachia Christinae Hance, Ligusticum Chuanxiong Hort, Bergamot, Jujube, Ginger, Viola Yedoensis Makino, Hawthorn.

\section{Model visualization}

Random forest is a classification and regression algorithm formed by multiple decision trees. The model is trained by samples to form a decision judgment for each traditional Chinese medicine component so as to achieve the purpose of prediction. Figure 2 shows part of the inference process 


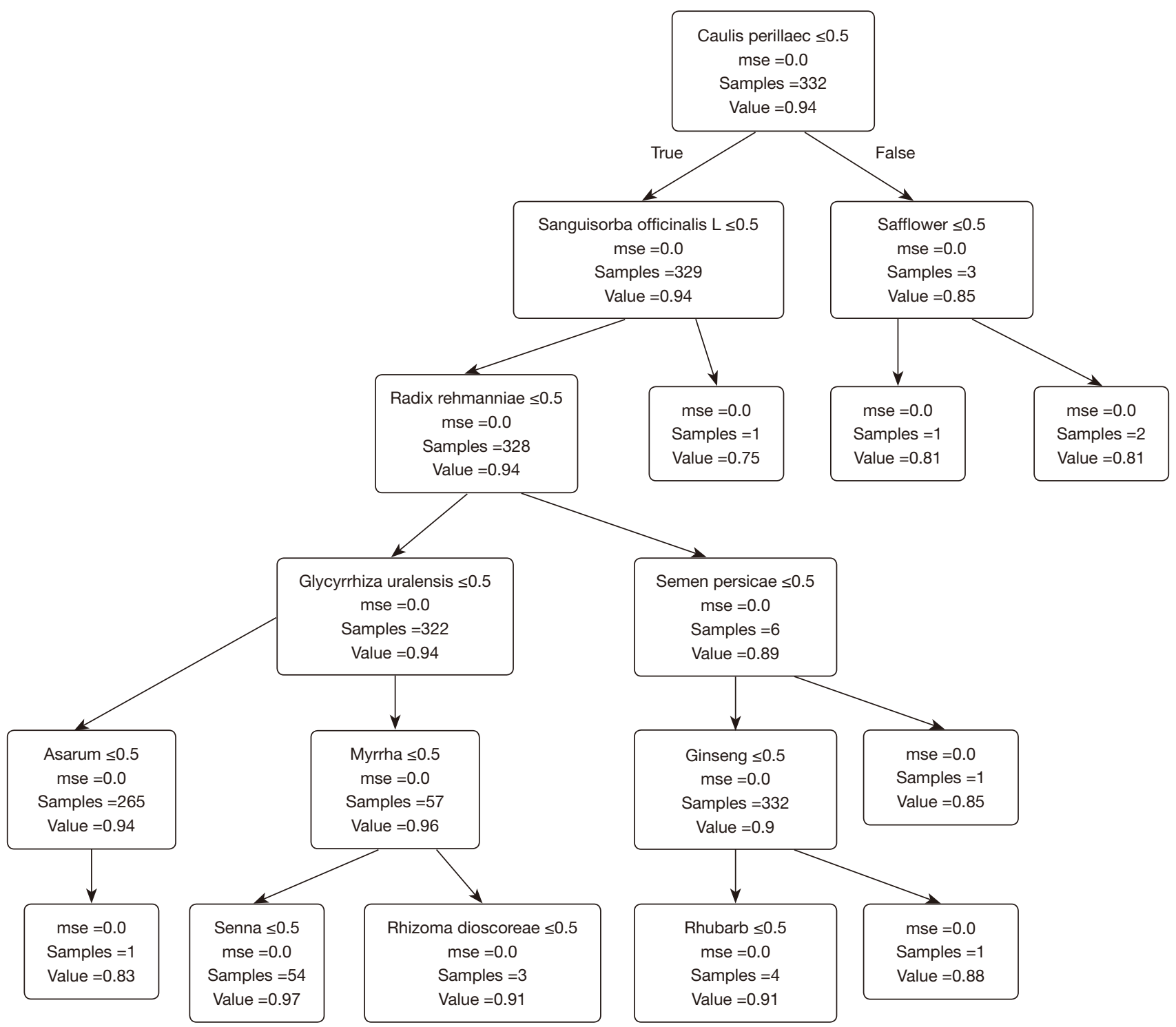

Figure 2 Partial visualization of a decision tree in the random forest model.

for one of the decision trees, where each leaf node is a judge that passes the judgment of whether to enter the corresponding lower-level node. A random forest consisting of multiple decision trees may be better in the prediction of prescription efficacy. We used $90 \%$ of the data for training and the remaining $10 \%$ of the data as a test set for prediction, and the results showed that the model displayed better prediction for the never seen test set, with a coefficient of determination $\mathrm{R}^{2}$ of 0.7318 , demonstrating that the model better reflected the relationship between the prescription and the effectiveness.

Based on the results of the random forest algorithm combined with the theory of Chinese medicine, we can conclude that soothing the liver, promoting qi, clearing heat, removing obstructions of organs, activating blood, and resolving stagnation are the treatment methods for AP.

\section{Discussion}

$\mathrm{AP}$ is a common acute abdominal disease, characterized by local inflammatory response of the pancreas and systemic inflammatory response syndrome (SIRS), which can be accompanied by organ dysfunction or failure in severe cases. The typical clinical presentation is an acute attack 
of epigastric pain with elevated serum amylase and lipase. About $85 \%$ of patients have acute interstitial edematous pancreatitis, characterized by an enlarged pancreas due to inflammatory edema. Approximately $15 \%$ of patients have necrotizing pancreatitis, which presents as moderately severe or severe AP with local or systemic complications or organ failure (10). There are multiple possible causes of $\mathrm{AP}$ and regional differences. Cholelithiasis is still the main cause of AP in China, followed by alcoholic AP. Currently, the incidence of hypertriglyceridemic pancreatitis (HTGP) is increasing year by year, and the incidence of the population is younger with more severe cases, surpassing alcoholic AP as the second leading cause, which requires further attention (11).

According to Chinese medicine, this disease belongs to the categories of "abdominal pain", "spleen heart pain", "syncope heart pain", "pancreatic disease", "diarrhea", and "convulsions and dehydration". The organs affected by the disease involve the liver, gallbladder, spleen, and stomach, and similar symptoms are recorded in the ancient classic Chinese medical text Ling Shu-Convulsive Diseases (12). $\mathrm{AP}$ is thought to be an obstruction of qi in the internal organs, with a combination of Yang Ming and Shao Yang evidence, and is attributed to depression, heat, stasis, knots, and syncope, often with diet, insects, and emotions as triggers. The pathogenesis of the disease is based on qi stagnation, damp-heat, blood stasis, and food stagnation in the middle jiao, which in turn leads to a loss of elevation in the spleen and stomach, a loss of drainage in the liver, and a loss of harmony and descent in the stomach. The cause of the disease includes liver depression and stagnation, dampness and heat accumulation, heat and toxicity, and stasis and heat as the 4 main pathogenic mechanisms of AP, which can cause the disease alone, but can also affect each other, blocking the internal organs of qi movement. When qi of internal organs is not clear, it leads to pain and the occurrence of the disease.

Researchers have shown that herbal medicine has the effect of regulating the secretory function of the pancreas in the treatment of AP. Zhang et al. (13) found that Dahuang Mudan decoction regulates pancreatic secretion by inhibiting amylase activity, and rutinoside in acacia rice $(14,15)$ enhances the secretion of trypsin and reduces serum amylase to achieve a better therapeutic effect in AP. Rhodopsin and baicalin alleviated the pathological process of AP to a certain extent by reducing serum amylase in rats with $\mathrm{AP}(16,17)$. It has also been shown that Chinese medicine can prevent and treat hyperamylasemia after endoscopic retrograde cholangiopancreatography (ERCP), an important treatment for pancreaticobiliary diseases (18).

Chinese medicines have the ability to modulate the inflammatory response. Inflammatory factors such as TNF- $\alpha$, IL-6, and IL-1 are important inflammatory mediators in the inflammatory response of extra-pancreatic organs in the late stages of AP (19). Studies have shown that rhubarb and aconite soup reduce the secretion of IL-6 and TNF- $\alpha$ by inhibiting the JAK2/STAT3 pathway (20), thereby downregulating NALP3 inflammatory vesicles (21). In mice with $\mathrm{AP}$, rhodopsin $(22,23)$ inhibited the release of inflammatory factors such as IL-1, IL- 8 , and TNF- $\alpha$. At the same time, rhodopsin reduced the proportion and activity of regulatory $\mathrm{T}$ cells in rats with severe AP, preventing further exacerbation of AP inflammation. Dahuang Mudan decoction blocks the HMGB1-TLR4 signaling pathway (24), and Chai Huang Qing Pancreatic Revitalizing Blood Granule regulates the expression of Foxp3 and ROR $\gamma \mathrm{t}$ transcription factors, both of which can regulate the inflammatory response (25) and prevent the exacerbation of AP.

Chinese medicines have a role in regulating apoptosis. Among the Chinese medicine recipes, Dahuang Mudan Tang can enhance pancreatic NO levels and iNOS activity to promote apoptosis (26). Qing Pancreas II can inhibit HMGB1-coupled TLR4 and reduce NF- $\kappa \mathrm{B}$ production (27). Da Cheng Qi Tang inhibits the release of sIL-2R and IL-6 in rats with severe AP (28), both of which can promote apoptosis. Among the traditional Chinese medicine monomers, Ligustrazine can inhibit the ERKMAPK and p38 pathways (29), and rhodopsin can promote the expression of Hes-1 and Notch-1 proteins (30), thus achieving the effects of promoting apoptosis, reducing the inflammatory response, and preventing further exacerbation of AP.

Chinese medicines have the effect of promoting microcirculation in the pancreas. In a rat model of AP (31), the difference between pancreatic microcirculation and venous capillary blood flow velocity was observed to be large by applying the fluorescence micro flow velocity quantification method. However, the difference between pancreatic microcirculation and venous capillary blood flow velocity in rats with AP was greatly improved after treatment with Dahuang Mudan decoction, indicating that Dahuang Mudan decoction can promote pancreatic microcirculation to achieve therapeutic effects. Chaishao Chengqi decoction (32) can adjust pancreatic microcirculation in patients with severe AP by improving the indicators of 
pancreatic blood viscosity and blood flow, and has achieved relatively good therapeutic effects.

Chinese medicines also have the effect of reducing calcium overload in the pancreatic glands. One of the necessary components in the development of AP is pancreatic damage due to calcium overload of the pancreatic follicles. Therefore, regulating calcium balance is a key tool in reducing the progression of AP. Zhang et al. (13) found that Dahuang Mudan decoction could reduce calcium overload in the pancreatic vesicles, inhibit their secretion of amylase, and prevent the progression of AP. Dong et al. (33) found that Panax ginseng total saponin could effectively inhibit Ca MKII- $\gamma$ protein expression in rat pancreatic tissues, thereby blocking calcium overload and reducing pancreatic injury. Carrasco et al. (34) reported that resveratrol induced abnormal calcium signaling in a model of AP, achieving a reduction in pancreatic injury.

In this study, in terms of the frequency of drug use, rhubarb topped the list, which is consistent with literature reviews by several scholars. Rhubarb was first found in the Divine Husbandman's Classic of the Materia Medica, with the effects of laxative and attacking accumulation, clearing heat and removing toxins, and promoting blood circulation and eliminating stasis. In Article 137 of the Treatise on Cold, it is stated that "when the pain is hard and cannot be approached from the heart down to the abdomen, Dachengqi decoction is the main remedy", and the main medicine of Dachengqi decoction is rhubarb. Modern pharmacological studies have confirmed that rhubarb has the following properties: it can inhibit the secretion of pancreatic enzymes, relax the biliary sphincter, and reduce pancreatic duct pressure; reduce the permeability of the gastrointestinal microvasculature caused by endotoxins, and inhibit intestinal bacterial translocation and endotoxin absorption; promote bile excretion, thereby inhibiting the absorption of endotoxins; inhibit a variety of bacteria, and maintain the balance of intestinal flora; improve intestinal paralysis and intestinal obstruction, and help improve the microcirculation of the pancreas; and significantly enhance the body's cellular immunity and promote the proliferation of lymphocytes (35).

The second most frequently used drug is Radix Bupleuri, which has the effect of reconciling Shaoyang and clearing heat and knots. The Divine Husbandman's Classic of the Materia Medica says "it is for the heart, abdomen, intestines, stomach and intestines." Saikosaponin can effectively inhibit the secretion of pancreatic protease, reduce the secretion of pancreatic juice, and reduce the probability of pancreatic infection $(36,37)$, which contains saikosaponin a and saikosaponin b has a strong inhibitory effect on $\mathrm{Na}^{+}, \mathrm{K}^{+}$and ATPase, which can effectively control pancreatic infection (38).

Fructus Aurantii Immaturus, Mirabilite, and Magnolia Officinalis are the third, fourth, and fifth most frequently used drugs, respectively. Fructus Aurantii Immaturus and Mirabilite have the function of treating abdominal distension, and Magnolia Officinalis has the function of resolving blood stasis and breaking up stagnation, and together with rhubarb, they form the famous Dachengqi decoction. Dachengqi decoction is an important prescription for treating hot knots and saving yin, and is highly effective in the treatment of AP. The high frequency of the use of these 4 drugs indicates that the condition of sthenic-fu and heat accumulated syndrome is one of the main symptoms of AP (39).

Of the first ten drugs, rhubarb, Radix Bupleuri, Fructus Aurantii Immaturus, Scutellaria Baicalensis Georgi, Radix Paeoniae Alba, and Pinellia Ternata make up the Dachaihu decoction. The Synopsis of Golden Chamber states that "if there is full pain under the heart by pressing, this is repletion, should be emitted, Dachaibu decoction is recommended." Dachaihu decoction reconciles Shaoyang, drains Yang Ming internally, treats the unfavorable pivot mechanism of Shaoyang and Yang Ming's internal organs caused by typhoid cold, and treats both the exterior and interior. Dachaihu decoction is effective in treating pancreatitis associated with liver depression and stagnation, dampness and heat in the liver and gallbladder, and heat and stagnation in the internal organs (40).

Among the top ten medications, the main medications of Dachengqi decoction, Dachaihu decoction, and Daxianxiong decoction were included. The use of these three classical and effective formulas follows the traditional Chinese medicine theory that "the six internal organs are used for the purpose of generalization", having the effect of relieving the liver and qi, clearing heat from the internal organs, and promoting blood circulation and resolving stasis (41).

The first place is rhubarb, which clears heat from the internal organs, cools blood and detoxifies toxins, promotes blood circulation and resolves blood stasis, and has a comprehensive therapeutic and regulatory role in the treatment of pancreatitis in many aspects.

The second in the model is Rhizoma Corydalis, which can invigorate blood, regulate qi, resolve stasis, and relieve pain. Its analgesic effect is the most powerful in traditional Chinese medicine, and the analgesic potency of total 
alkaloids of Rhizoma Corydalis can be up to $40 \%$ of that of morphine, so it has good antispasmodic and analgesic effects. It can activate and promote the secretion of antiinflammatory factors, plays an antibacterial and antiinflammatory role, and at the same time has analgesic and immunomodulatory effects (42). Rhizoma Corydalis, together with rhubarb and other traditional Chinese medicines, can be used to address the complexity of AP pathology in a multi-targeted and comprehensive manner. Therefore, Rhizoma Corydalis ranks second in terms of efficacy.

This is followed by Pinellia ternata, Magnolia officinalis, Radix Paeoniae Alba, Radix Bupleuri, Scutellaria baicalensis Georgi, Curcuma longa L, and Fructus aurantii immaturus, covering the medicinal composition of Dachaihu decoction, as well as the main components of Dachengqi decoction, Chaiqin Chengqi decoction, and Chaishao Chengqi decoction. Eight randomized controlled trials after metaanalysis showed that the treatment of AP with Dachai $\mathrm{Hu}$ Tang significantly reduced the inflammatory response and the levels of the inflammatory factors IL- 6 and TNF- $\alpha$, thus improving the therapeutic effect of patients with $\mathrm{AP}(43,44)$.

Traditional Chinese medicine in the treatment of AP mainly acts on the regulation of pancreatic secretion function, inflammatory responses, and apoptosis, among other processes, and there is an interconnection between these mechanisms. Dead pancreatic follicular cells may affect the local microcirculation of the pancreas, which in turn may lead to aggravation of the inflammatory response, resulting in a pathological interactive amplification effect, and traditional Chinese medicine can treat AP in multiple ways (45).

The coefficient of determination $\mathrm{R}$-squared score is usually used as an indicator to evaluate the model's performance. The closer the coefficient of determination is to 1 , the greater the association between the drug and effectiveness. In our random forest model, the R-squared score reached 0.8021 which showed that our model fit the data very well. In order to avoid over-fitting of the model and to prove that the model has generalization, we trained the model with $90 \%$ of the data and tested its performance on the other $10 \%$ of the data. The result showed that the $\mathrm{R}$-squared score achieved 0.7318 on the test set which indicates that the confidence of the results predicted by the model is high. Therefore, the compounds recommended by the model are likely to be effective in the treatment of AP.

The random forest algorithm is an effective machine learning algorithm that integrates multiple decision trees to improve accuracy through integrated learning, and can be used in the study of classification and regression problems. The random forest algorithm can take into account the interaction between variables and the influence of various factors on the results, and score the importance (9).

In this paper, the random forest algorithm was used to explore the medication rules of treatment for AP, which provides a new method for exploring medication rules, and the results are in line with the classical theory and clinical practice of traditional Chinese medicine.

The clinical selection of prescriptions and medicines in traditional Chinese medicine should be based on diagnosis and treatment. Combining traditional Chinese medicine theory with artificial intelligence will enable more precise treatment and improve clinical efficacy, while also providing a valuable source of prescriptions for the development of new drugs. It is believed that the development of modern science and technology can strongly promote the advancement of traditional Chinese medicine in modern precision medicine, as well as the development of traditional Chinese medicines.

\section{Acknowledgments}

Funding: Project supported by the Shanghai Specialized Research Fund for Integrated Chinese and Western Medicine in General Hospitals (ZHYY-ZXYJHZX-201914).

\section{Footnote}

Conflicts of Interest: All authors have completed the ICMJE uniform disclosure form (available at https://dx.doi. org/10.21037/apm-21-2505). The authors have no conflicts of interest to declare.

Ethical Statement: The authors are accountable for all aspects of the work in ensuring that questions related to the accuracy or integrity of any part of the work are appropriately investigated and resolved.

Open Access Statement: This is an Open Access article distributed in accordance with the Creative Commons Attribution-NonCommercial-NoDerivs 4.0 International License (CC BY-NC-ND 4.0), which permits the noncommercial replication and distribution of the article with the strict proviso that no changes or edits are made and the original work is properly cited (including links to both the formal publication through the relevant DOI and the license). 
See: https://creativecommons.org/licenses/by-nc-nd/4.0/.

\section{References}

1. Arji G, Safdari R, Rezaeizadeh H, et al. A systematic literature review and classification of knowledge discovery in traditional medicine. Comput Methods Programs Biomed 2019;168:39-57.

2. Zhang H, Ni W, Li J, et al. Artificial Intelligence-Based Traditional Chinese Medicine Assistive Diagnostic System: Validation Study. JMIR Med Inform 2020;8:e17608.

3. Wang Y, Shi X, Li L, et al. The Impact of Artificial Intelligence on Traditional Chinese Medicine. Am J Chin Med 2021;49:1297-314.

4. Han K, Zhang L, Wang M, et al. Prediction Methods of Herbal Compounds in Chinese Medicinal Herbs. Molecules 2018;23:2303.

5. Wang Z, Zhang L, Wang M. Approaching High-Accuracy Side Effect Prediction of Traditional Chinese Medicine Compound Prescription Using Network Embedding and Deep Learning. IEEE ACCESS 2020;8:82493-9.

6. Xie J, Wang N, Ding R. Association learning of Chinese herbal medicines and disease treatment efficacy. Int J Prod Res 2019;57:683-702.

7. Zhu G. Traditional Chinese medicine. Beijing: Tsinghua University Press. 2021;171:112730. doi: 10.1016/ j.bios.2020.112730.

8. Medicine SAoC, Committee. CMMW. Chinese Materia Medica. Shanghai: Shanghai Science and Technology Press, 1999.

9. Wen T, Liu B, Zhang Y. Risk factors for unplanned hospital readmission within 31 days in patients with ischemic stroke: a randomized forest model. Chinese Journal of Evidence-Based Medicine 2019;19:532-8.

10. Association PSGotCM. Guidelines for the diagnosis and management of acute pancreatitis (2014). Chinese Journal of Practical Surgery 2015;35:4-7.

11. Gastroenterology PDGCSo, Diseases ECoCJoP, Gastroenterology ECoCJo. Guidelines for the diagnosis and treatment of acute pancreatitis in China (2019, Shenyang). Chinese Journal of Pancreatic Diseases 2019;19:321-30.

12. Unknown. Ling Shu Jing. Beijing: People's Health Publishing House, 2016.

13. Zhang Y, Shu C, Cai X, et al. Effects of the group formula of Dahuang Mudan Tang on the secretory function of glandular alveolar cells in rats with acute pancreatitis model. Experimental Animal Science 2014;31:26-9.
14. Aruna R, Geetha A, Suguna P. Rutin modulates ASC expression in NLRP3 inflammasome: a study in alcohol and cerulein-induced rat model of pancreatitis. Mol Cell Biochem 2014;396:269-80.

15. Abreu FF, Souza AC, Teixeira SA, et al. Elucidating the role of oxidative stress in the therapeutic effect of rutin on experimental acute pancreatitis. Free Radic Res 2016;50:1350-60.

16. Li H, Zhang C, Zhang S. Study on the effect of the combination of rhodopsin and baicalin on the Akt/Nrf2 pathway in rats with acute pancreatitis model. Chinese Pharmacy 2018;29:1754-9.

17. Pan H. Experimental study on the effect of raw rhubarb on the expression of blood amylase and TNF- $\alpha$ in a rat model of acute pancreatitis. Dali: Dali College, 2014.

18. Liao Y, Cui L, Zhang C. Progress of research on the prevention of hyperamylasemia and pancreatitis after ERCP surgery with Chinese medicine. Journal of Modern Integrative Chinese and Western Medicine 2016;25:225-8.

19. Xu D, Xun J. Research progress of severe acute pancreatitis with persistent inflammation-immunosuppressioncatabolic syndrome. Chinese Journal of Practical Diagnosis and Therapy 2018;32:725-8.

20. Wu L, Cai B, Liu X, et al. Effects of rhubarb and aconite soup-containing serum on JAK2/STAT3 signaling pathway in peritoneal macrophages of mice with severe acute pancreatitis. Chinese Herbal Medicine 2013;44:3195-9.

21. Wang P. Expression of NALP3 inflammasome in lung injury of severe acute pancreatitis in rats and experimental study on the intervention effect of rhubarb and azuki soup. Zunyi: Zunyi Medical College 2017.

22. Yao WY, Zhou YF, Qian AH, et al. Emodin has a protective effect in cases of severe acute pancreatitis via inhibition of nuclear factor- $\mathrm{\kappa} B$ activation resulting in antioxidation. Mol Med Rep 2015;11:1416-20.

23. Zhang Z. Study on the mechanism of action of rhodopsin in the treatment of severe acute pancreatitis. Tianjin: Tianjin Medical University, 2015.

24. Song B. Effect of rhubarb and peony soup on HMGB1_ TLR4 expression in pancreatic tissue of rats with acute pancreatitis. Lanzhou: Gansu Agricultural University, 2018.

25. Zhu H. The efficacy of Chai Huang Qing Pancreatic and Blood Activating Granules on patients with severe acute pancreatitis and its effect on Treg and Th17-related factors. Luzhou: Southwest Medical University, 2018.

26. Zhang Y, Wang Y, Zhang Y, et al. Effects of the group formula of Dahuang Mudan Decoction on apoptosis of pancreatic cells in rats with acute pancreatitis. Jilin Chinese 
Medicine 2014;34:982-4.

27. Zhou M. The effect of Qing Pancreas II on apoptosis of glandular alveolar cells in rats with severe acute pancreatitis and exploration of the mechanism. Zunyi: Zunyi Medical College, 2017.

28. Zhang M, Du J. Study of Da Cheng Qi Tang on the induction of apoptosis of pancreatic alveolar cells by serum IL-6 sIL-2R in rats with acute severe pancreatitis. Modern Medicine and Health 2018;34:352-4, 7.

29. Chen J, Chen J, Wang X, et al. Ligustrazine alleviates acute pancreatitis by accelerating acinar cell apoptosis at early phase via the suppression of p38 and Erk MAPK pathways. Biomed Pharmacother 2016;82:1-7.

30. Shi Z, Fang K, Sun J, et al. Interventional effect of rhodopsin on apoptosis of pancreatic cells in rats with severe acute pancreatitis. Chinese general medicine 2017;15:1830-4.

31. Pan J, Zhang Y, Wang Y, et al. Effects of the group formula of Dahuang Mudan Decoction on pancreatic microcirculation in rats with acute pancreatitis model. Experimental Animal Science 2016;33:48-51.

32. Li X, Ke C. Effect of self-prepared Chinese herbal treatment on pancreatic microcirculatory indexes in patients with severe pancreatitis. World Chinese Medicine 2018;13:2995-8.

33. Dong W, Zhou Y, Liu Z, et al. Effect of Panax notoginseng total saponin on CaMKII- $\gamma$ expression in calcium overload in acute severe pancreatitis in rats. unnan Journal of Traditional Chinese Medicine 2018;39:67-70.

34. Carrasco C, Holguín-Arévalo MS, Martín-Partido G, et al. Chemopreventive effects of resveratrol in a rat model of cerulein-induced acute pancreatitis. Mol Cell Biochem 2014;387:217-25.

35. Weng W. Effectiveness of Chinese rhubarb in the treatment of severe acute pancreatitis. China Modern Drug Application 2020;9:194-5.

36. Sheng H. Logistic regression analysis of the prognostic factors of hepatobiliary dampness and fever in acute

Cite this article as: $\mathrm{Lu}$ WW, Chen X, Ni JL, Cai WJ, Zhu SL, Fei AH, Wang XS. Study on the medication rule of traditional Chinese medicine in the treatment of acute pancreatitis based on machine learning technology. Ann Palliat Med 2021;10(10):10616-10625. doi: 10.21037/apm-21-2505 biliary pancreatitis at different times of traditional Chinese medicine intervention. The World of Chinese and Western Medicine 2019;14:1435-8.

37. Cheng J. Evaluation of the efficacy of nasal feeding with Chinese herbal enema in the treatment of acute pancreatitis associated with liver depression and stagnation and hepatobiliary dampness and heat. Zhejiang Journal of Chinese Medicine 2020;5 5:181-3.

38. Shi X, Zeng H. Study on the clinical efficacy of enema with Chai Hu Shu Hepatic San on acute pancreatitis. Journal of Sichuan North Medical College 2019;34:619-21.

39. Jia Y. Clinical study on the characteristics of acute pancreatitis in Chinese medicine. Journal of Modern Integrative Chinese and Western Medicine 2018;27:343-6.

40. Zhuo Y. Progress in the study of the mechanism of action of traditional Chinese medicine compound for the treatment of acute pancreatitis. Chinese Journal of Integrative Medicine and Surgery 2019;25:394-8.

41. Yan L. Progress in the treatment of acute pancreatitis by Chinese herbal medicine. Asia-Pacific Traditional Medicine 2019:207-9.

42. He X, Zhang J, Zhang M. Progress in the study of chemical composition, pharmacological activity and toxic side effects of Rhizoma Corydalis. Shanghai Journal of Traditional Chinese Medicine 2017;51:97-100.

43. Yang X, Hu Z. Meta-analysis of the clinical efficacy of Dachaihu Decoction in acute pancreatitis. Modern Practical Medicine 2017;29:29-33.

44. Yang B, Li B. Observation on the efficacy of preserved enema with Da Chai Hu Tang on acute pancreatitis and prevention of intestinal flora dysbiosis. Chinese Traditional Chinese Medicine Emergency 2019;28:2035-7.

45. Sun $W$. Research progress on the mechanism of Chinese medicine for acute pancreatitis. Chinese Patent Medicine 2019;41:1932-5.

(English Language Editor: C. Betlzar) 